\section{Poder y dinero en la enfermedad de Chagas: una histórica omisión}

\author{
Power and money in Chagas \\ disease: a historical omission
}

\author{
Storino, Rubén ${ }^{1}$ \\ ${ }^{1}$ Doctor en Medicina. Profesor en Ciencias Médicas. Adjunto \\ Cátedra de Salud Pública, Facultad de Ciencias Médicas, \\ Universidad Nacional de La Plata, Argentina. \\ r_storino@yahoo.com.ar
}

Comentario sobre: Zabala JP. La enfermedad en su laberinto: avances, desafíos y paradojas de cien años del Chagas en Argentina. Salud Colectiva. 2012;8(Supl 1):S9-S21.

En el artículo "La enfermedad en su laberinto: avances, desafíos y paradojas de cien años del Chagas en la Argentina" (1), Juan Pablo Zabala hace una interesante síntesis sobre diferentes aspectos biológicos, políticos, sociológicos y técnicos.

Sin embargo omitió referirse específicamente a dos aspectos que a mi entender son fundamentales para comprender por qué la enfermedad de Chagas aún sigue siendo un problema de salud pública no resuelto en la Argentina a diferencia de otros países vecinos como Uruguay, Chile y Brasil donde se logró controlarla. Estos aspectos son:

1. El poder de un grupo hegemónico de profesionales que manejó las decisiones y el control del Chagas durante los últimos 40 años en la Argentina, específicamente desde el Estado, que abarcó desde la lucha contra el vector hasta el otorgamiento de subsidios de investigación.

2. Los intereses económicos de un sector allegado a ese grupo de poder que privilegió las inversiones y el destino del dinero en dos aspectos que, si bien son importantes, no son los únicos en el Chagas: el desarrollo de reactivos serológicos y los insecticidas.
Para entender este análisis, debemos asumir previamente que el paradigma de la problemática de la enfermedad de Chagas excede el marco bio-psico-social, dado que involucra factores de poder político y económico, por lo cual pasa a constituirse no solo en una tradicional enfermedad de la pobreza, sino en un paradigma de los mecanismos de ocultamiento y exclusión como forma de discriminación social y laboral.

En esta realidad intervienen varios actores: el Estado, los investigadores, los médicos, los portadores serológicos chagásicos, los enfermos chagásicos, la sociedad, los medios de comunicación y la industria farmacéutica.

Todos contribuyen, en alguna medida, a mantener cierta indiferencia en solucionar definitivamente el problema. El marco dominante es el intento de cada sector por privilegiar sus propios intereses, de manera tal que se excluye una tarea comunitaria conjunta en la que los saberes y poderes están al servicio de los indigentes, marginados y desposeídos sociales que sufren esta enfermedad.

El Estado al ocuparse a través de sus funcionarios de minimizar el problema, los investigadores con su actitud de priorizar sus becas y subsidios, los médicos al desinteresarse por una enfermedad que afecta a pacientes pobres, los portadores serológicos chagásicos al ocultar su situación por sus experiencias de exclusión laboral, los enfermos chagásicos desprotegidos del sistema de seguridad social, la sociedad indiferente, los medios de comunicación ausentes, y la industria farmacéutica al desertar de la investigación de nuevos fármacos por la escasa rentabilidad, forman el abanico del fracaso, constituyéndose en factores determinantes de la perpetuación de esta enfermedad (2).

Para comprender estos procesos debemos realizar, además, un análisis histórico por el cual se llegó a esta situación actual.

Salvador Mazza, el redescubridor de la enfermedad de Chagas cometió un error al trasladar la Misión de Estudios de Patología Regional Argentina (MEPRA) desde Jujuy a Buenos Aires, poco antes de su muerte acaecida en México en forma repentina, en 1946. De ese modo, la enfermedad de Chagas perdió la oportunidad de tener 
un centro de investigación de excelencia en terreno y de poder constituirse en un referente internacional sobre el tema como aconteció con el Instituto Oswaldo Cruz en Brasil.

De tal manera que todo el Chagas quedó para ser investigado desde Buenos Aires, aunque en estado de letargo, hasta que comenzó su despegue a partir de los años 1960 y 1970 con la creación del Instituto Nacional de Parasitología "Dr. Mario Fatala Chabén" y el Programa Nacional de Chagas con base operativa en Córdoba. En este marco se instaló un grupo de poder hegemónico que dominó prácticamente todo el abanico del Chagas y que en alguna medida lo sigue haciendo en los últimos cuarenta años.

Este grupo, con anclaje en el Estado Nacional, que incluso sobrevivió a todos los gobiernos independientemente de que fueran militares, peronistas o radicales, estaba constituido por funcionarios públicos, profesionales de las distintas ciencias de la salud, investigadores básicos y técnicos, cuyo modelo de abordar el Chagas consistía en priorizar el desarrollo de reactivos serológicos y de insecticidas. Este modelo se ejecutó con una concepción vertical, no solo en la estructura del poder, sino bajo una dirección hegemónica desde Buenos Aires hacia el interior del país. De ese modo, se dejaron de lado otros paradigmas como la descentralización del poder en manos de las provincias e incluso de los municipios, la atención integral de pacientes, la provisión de medicamentos cardiológicos a los pacientes con cardiopatía chagásica, sin entrar en otros aspectos más complejos como el tema de la educación, la vivienda y la inserción laboral de los que tienen Chagas. Si bien el modelo verticalista centralizado en Buenos Aires, con la mirada y el presupuesto en la lucha contra la vinchuca y la detección de serologías positivas para Chagas logró algunos resultados importantes, no pudo erradicar aún la transmisión de la enfermedad en varias provincias. Entre 1962 y 1991 este modelo significó un gasto estatal de 500 millones de dólares que se distribuyeron en sueldos, viáticos e insumos (3). A partir de entonces el presupuesto del Chagas quedó incluido junto a otras enfermedades endémicas, lo que impide saber cuál es el gasto real en Chagas en las últimas décadas en la Argentina.
Del análisis de estas cifras podemos señalar que es demasiado lo que se invirtió y poco lo que quedó como anclaje indispensable para acciones sostenidas. La pregunta que surge es: ¿por qué no se invirtió en capacitar y empoderar a las 200 comunidades que aún están en riesgo de transmisión vertical para que ellas mismas ejecuten las acciones contra el Chagas? Una respuesta sería: para que el mismo grupo siga manejando el poder y el dinero de manera vertical y centralizada.

Tampoco se hizo nada para desarrollar económicamente aquellos pueblos de zonas endémicas con transmisión vectorial, en los que el progreso aún no llegó.

Durante años, en los grupos profesionales independientes que trabajan en Chagas y que se han sentido marginados por el poder hegemónico se acuñó la frase "son más los que viven del Chagas que los que se curan de Chagas".

Si se analizan las decisiones políticas y económicas y las redes de poder conformadas por un elenco estable de funcionarios del Estado Nacional, junto a algunos profesionales de la salud y varios investigadores básicos para el financiamiento no solo de las acciones de control sino para el desarrollo de técnicas de diagnóstico, de biosensores para la detección de vinchucas y de investigaciones sobre supuestos marcadores biológicos del daño cardíaco, podemos afirmar que se dilapidó demasiado dinero, con el agravante de casos de denuncias penales por corrupción aunque con sobreseimiento como es habitual en Argentina.

El mayor problema es que ese dinero no le Ilegó a la gente que padece Chagas para modificar la multidimensionalidad de esta enfermedad, dado que sigue debatiéndose en la pobreza del rancho como vivienda estable, sin educación y en las peores condiciones laborales.

El avance en el conocimiento sobre los múltiples aspectos de la enfermedad de Chagas tuvo como resultado una parcelación del saber con claros beneficios para los investigadores básicos que pudieron acceder a cuantiosos subsidios, a diferencia de los pacientes involucrados en esos mismos estudios que solo recibieron las gracias por facilitar las muestras de sangre y posar para las fotos.

Históricamente, los que padecen Chagas han sido reducidos a una imagen testimonial, sin tener injerencia en las iniciativas, en las decisiones o en los gastos del dinero destinado al Chagas. 
La solución a esta histórica injusticia radica en un cambio de paradigma al mejor estilo de la revolución científica planteada por Kuhn (4).

Para esto es necesario realizar dos modificaciones sustanciales:

1. Reemplazar el paradigma actual del poder hegemónico vertical estatal y de profesionales e investigadores aliados por el empoderamiento de los pacientes con Chagas $y$, en especial, aquellos que habitan en las comunidades postergadas de zonas endémicas, aplicando el Modelo Ecosistémico de la Salud con plena injerencia en las decisiones políticas y económicas (5).

2. Reemplazar el paradigma lineal actual, cuyos modelos más empleados son el diagnóstico serológico y el uso de insecticidas por el paradigma de la complejidad según Morín (6) que reconoce la multicausalidad y multidimensionalidad tal como se da en la problemática del Chagas.

Por lo tanto, debe encararse una tarea conjunta, en la que las acciones se traduzcan en medidas concretas que fortalezcan el desarrollo y la participación de la comunidad, involucrando también a otras ciencias como la antropología, la sociología, la ecología, la psicología, la política y la economía, abarcando todos los niveles de prevención, enfocando la atención médica integral del paciente chagásico, implementando centros de estudio y control de la enfermedad de Chagas en todos sus aspectos, especialmente en la inserción laboral. Debemos modificar la situación de marginación y olvido que padecen millones de pacientes con Chagas víctimas de una enfermedad de la pobreza, agravada por su ocultamiento y por las relaciones de poder e intereses económicos donde se benefician unos pocos que no tienen Chagas en perjuicio de millones que sí tienen Chagas.

\section{REFERENCIAS BIBLIOGRÁFICAS}

1. Zabala JP. La enfermedad en su laberinto: avances, desafíos y paradojas de cien años del Chagas en Argentina. Salud Colectiva. 2012;8(Supl 1):S9-S21.

2. Storino R. La cara oculta de la enfermedad de Chagas. Revista de la Federación Argentina de Cardiología. 2000;29(1):31-44.

3. Segura E. El control de la enfermedad de Chagas en la República Argentina. En: El control de la enfermedad de Chagas en los países del Cono Sur de América [Internet]. OPS; 2002 [citado 25 sep 2012]. Disponible en: http://www.paho.org/Spanish/AD/DPC/CD/dch-historia-incosur.htm

4. Kuhn T. La estructura de las revoluciones científicas. México DF: Fondo de Cultura Económica; 1980.

5. Possas CA. Social ecosystem health: confronting the complexity an emergence of infectious diseases. Cadernos de Saúde Pública. 2001;17(1):31-41.

6. Morin E. Introducción al pensamiento complejo. Madrid: Gedisa; 2003.

\section{FORMA DE CITAR}

Storino R. Poder y dinero en la enfermedad de Chagas: una histórica omisión. [Debate]. Salud Colectiva. 2012;8(Supl 1):S23-S25.

\section{Resonancias sociales y políticas del estudio social de una enfermedad}

\section{Social and political reverberations in the social study of a disease}

\footnotetext{
Stagnaro, Adriana Alejandrina ${ }^{1}$

${ }^{1}$ Doctoranda en Antropología Social. Investigadora, Instituto de Ciencias Antropológicas. Profesora Adjunta, Facultad de Filosofía y Letras, Universidad de Buenos Aires, Argentina. astagnaro@uolsinectis.com.ar
}

Comentario sobre: Zabala JP. La enfermedad en su laberinto: avances, desafíos y paradojas de cien años del Chagas en Argentina. Salud Colectiva. 2012;8(Supl 1):S9-S21.

Con un enfoque multifacético de la enfermedad de Chagas y su expansión por algo más de un siglo en la Argentina, Juan Pablo Zabala (1) aporta ganancias heurísticas a la reflexión sobre los enfoques con los cuales se ha delineado tradicionalmente su definición.

La discusión centrada en las tensiones operantes en la configuración de la enfermedad y 